\title{
Time to viral load suppression and its associated factors in cohort of patients taking antiretroviral treatment in East Shewa zone, Oromiya, Ethiopia, 2018
}

\author{
Jemal Hassen Ali ${ }^{*}$ a and Tewodros Getinet Yirtaw
}

\begin{abstract}
Background: A key goal of Antiretroviral Treatment (ART) is to achieve and maintain durable viral suppression. Thus, the most important use of viral load measurement is to monitor the effectiveness of therapy after initiation of ART. The main objective of the study was to determine the time for virological suppression and its associated factors among people living with HIV taking antiretroviral treatments in East Shewa Zone, Oromiya, Ethiopia.

Methods: Patients diagnosed with Human Immunodeficiency Virus presenting to the study health centers between October 3, 2011 and March 1, 2013 were included in the study given the following criteria: age 18 years or greater, eligible to start ART. All patients with baseline viral load measurements were included in the study. Interaction between explanatory variables with the response variable was analyzed by using cross tab features of (Statistical Package for the Social Sciences) SPSS, International Business Machines (IBM) Inc. Significance group comparison was done by Kaplan Meier log-rank test. Cox proportional hazard model was used to select significant factors to the variability between groups.

Result: Plasma viral load was suppressed below the detection level in $72 \%$ of individuals taking a different regimen of ART. The median Human Immunodeficiency virus (HIV)-1 plasma viral load in the cohort was estimated to be log 5.3111 copies $/ \mathrm{ml}$. The study observed Survival curve difference in the category of marital status ( $p$-value 0.023) and baseline cluster of differentiation 4 (CD4) value ( $p$-value 0.023 ). The estimated median time to Plasma Viral Load (PVL) suppression was 181 days (Cl: 140.5-221.4) with the age group of 30-39years having minimum time to achieve suppression with 92 days (Cl: 60.1-123.8) and the maximum time required to reach the level was found among the age group between 50 and 59 years.
\end{abstract}

Conclusion: The study found that the estimated time to achieve PVL after taking ART to be 181 days. Factors affecting time to suppression level were marital status and baseline CD4.

Keywords: Viral load, ART, Ethiopia, Suppression, HIV

\footnotetext{
* Correspondence: zaweyb@yahoo.com

Department of Public Health, Ethiopian Field Epidemiology Training

Program, St. Paul's Hospital Millennium Medical College, Addis Ababa

Ethiopia
}

(c) The Author(s). 2019 Open Access This article is distributed under the terms of the Creative Commons Attribution 4.0 International License (http://creativecommons.org/licenses/by/4.0/), which permits unrestricted use, distribution, and reproduction in any medium, provided you give appropriate credit to the original author(s) and the source, provide a link to the Creative Commons license, and indicate if changes were made. The Creative Commons Public Domain Dedication waiver (http://creativecommons.org/publicdomain/zero/1.0/) applies to the data made available in this article, unless otherwise stated. 


\section{Background}

Human immunodeficiency virus (HIV) is a virus transmitted through different body fluids such as blood and semen. The virus attacks the body's immune system of which is supposed to protect our body from attack [1]. Acquired immunodeficiency syndrome (AIDS) is a condition caused by the human immunodeficiency virus (HIV) [2].

United nation set goal to ending the AIDS epidemic by 2030 as part of the sustainable development goals (SDG) [3, 4]. Sustainable development goals set target in which: By 2020, 90\% of all people living with HIV will know their HIV status, 90\% of all people with diagnosed HIV infection will receive sustained antiretroviral therapy and $90 \%$ of all people receiving antiretroviral therapy will have viral suppression. When this goal is achieved, it is believed that at least $73 \%$ of all people living with HIV worldwide will be virally suppressed. Mathematical modeling suggests that achieving the above mentioned SDG targets by 2020 will enable the world to end the AIDS pandemic by $2030[5,6]$. Determination of Viral load in the patient plasma is the most important indicator of response to ART. It should be measured in all HIVinfected patient's plasma at entry into care, at the initiation of ART, and regularly monitoring of progress [7]. In this study, plasma viral load suppression was classified as $<1000$ copies/ml as suppressed and plasma viral load of $>1000$ copies $/ \mathrm{ml}$ was classified as an unsuppressed plasma viral load level in the patient plasma. Viral load (HIV-RNA copies/ml of blood plasma) is used as a surrogate marker for disease progression [8]. The main objective of this study was to determine the time to virological suppression and its associated factors among people living with HIV taking antiretroviral treatments in East Shewa zone, Oromiya, Ethiopia, 2018.

\section{Methods}

\section{Settings}

The study was conducted in East Shewa zone Oromiya Region, Ethiopia in 2018. Those patients who take ART treatment in five Health centers in East Shewa zone were included.

The study was conducted on patients who were enrolled between October 3, 2011 and March 1, 2013.

\section{Study design}

Retrospective cohort study design was used to conduct the study.

\section{Source population}

The source population was all people with confirmed HIV positive status in East Shewa Zone, Oromiya region Ethiopia.

\section{Study population}

HIV patients who have started ART service in East Shewa zone between October 3, 2011 and March 1, 2013 with at least two consecutive viral load tests were included.

\section{Sample size determination and sampling}

In this study, 243 study participants who have baseline viral load measurement were included in the study. All patients in the cohort with baseline Plasma viral load were included in the study.

\section{Data collection tools \& procedures}

The study used secondary data from a long-running community-recruited prospective cohort of patients living with HIV. HIV Plasma Viral load measurement was taken at enrollment of patient to the study (Baseline), and then after the first month, third month, six months and twelve months and eighteen months of ART treatment. Trained clinicians at the start of the study collected demographic and clinical data, including physical examination details by using structured questionnaires. Interview was also used to collect data from patients. After informed consent, data were collected and checked for completeness.

\section{Laboratory analysis}

All laboratory analyses, except Liquid cultures for Tuberculosis (TB), were performed at Adama Public Health Research and Referral Laboratory Center. CD4 cell counts were analyzed by using BD FACSCalibur cytometer (Becton Dickinson, San Jose, CA). Sputum and Fine Needle Aspirate (FNA) samples were analyzed with direct smear microscopy using Ziehl-Neelsen staining and Xpert MTB/RIF (Cepheid, Sunnyvale, CA) for polymerase chain reaction (PCR). Liquid cultures for $\mathrm{TB}$ were performed at International Clinical Laboratories, Addis Ababa, using a BACTEC MGIT 960 (BD Diagnostics, Franklin Lakes, NJ). Plasma HIV-RNA levels were determined using Abbott Real- Time HIV-1 assay (Abbott Molecular Inc., Des Plaines, IL) with a detection limit of 40 copies/ml. External quality assurance of the regional laboratory is regularly performed by the Center for Disease Control and Prevention (Atlanta, GA) [7].

\section{Eligibility criteria}

Human immunodeficiency virus (HIV)- positive patients coming to the study health facilities between October 3, 2011 and March 1, 2013 were eligible for inclusion that met the following criteria: age 18 years or above and residency in the catchment area of any of the study sites. This cohort has since been continuously followed for well over 8 years. Those patients who have started Antiretroviral Treatment (ART) constitute the study population, with 
follow-up data collected until data abstraction on December 31, 2013 [7].

\section{Exclusion criteria}

Age less than 18 years old, with current or previous ART, as well as patients on ATT for more than 2 weeks before inclusion in this study were excluded from this study.

\section{Data quality}

Training was given for two data clerks on data management of secondary data obtained from the previous study. Pretest of data collection tools was done before the actual data collection procedure. Double-entry of data made by using EPI INFO 7 to minimize error in data management and analysis was done by SPSS version 21.

\section{Data analysis}

Once data is collected, it was analyzed descriptively to determine the demographic characteristics and distribution of time to PVL. Time to undetectable viral load level was calculated by using Kaplan-Meier to estimate the distribution of PVL in time, and the difference between the survival curves was evaluated with the use of the log-rank test, which is useful for comparing potentially unequal follow-up times of study participants. Cox proportional-hazards regression analysis used to relate covariates with response variable with $95 \%$ confidence intervals.

\section{Result}

\section{Study participant characteristics}

Study participants were recruited from Adama, Dhera, Geda, Modjo and Wolenchiti health centers taking ART and selected according to eligibility criteria. The minimum age included in the study was 18 years and the maximum age was 69 years old. Forty-five percent of the participant's age was between 20 and 29 years old and the minimum category was observed in $<20$ years old with $1.6 \%$. The sex distribution of the study participant was $41 \%$ male and $59 \%$ female participants. Most of the study participants $(41 \%)$ were married but widowed participants represented only $10 \%$ of the participants. At enrollment, $13 \%$ of the study participants have a plasma viral load of less than 10,000 copies/ $\mu$ l. plasma viral loads were suppressed in $72 \%$ of individuals taking a different regimen of ART treatment. The median HIV-1 plasma viral load in the cohort was log 5.3111 copies $/ \mathrm{ml}$. Nine percent of participants' complete higher education while $36 \%$ of them were illiterate. Study participant's nutritional status was also assessed by the study. Six participants (2.46\%) were classified as having acute malnutrition while $71(29.2 \%)$ Participants classified as having moderate malnutrition and the rest 166 (68.3\%) were classified as normal. The Baseline CD4 count is the value of a CD4 count of the study participants determined before initiating ART. It is determined when the study participant enrolled in the study. Based on the data 140(57.6\%) of the participants have Baseline CD4 (BCD4) count of $<200$ cells/ $\mu$ l, 77(31.7\%) have BCD4 count 200-350 cells/ $\mu$ land 26(10.7\%) have BCD4 count of $>350$ cells $/ \mu$ l. Tuberculosis disease screened among participants before enrollment and 189(77.8\%) were positive for Mycobacterium tuberculosis (MTB) by Acid Fast Bacilli (AFB) light microscopy smearing and Liquide and solid culture Mycobacteria Growth Indicator Tube (MGIT) and Löwenstein-Jensen (LJ) respectively. At enrollment, $77 \%$ of study participants without Tuberculosis (TB) disease were found to have BPVL of $<1000$ copies/ $\mathrm{ml}$, while $84 \%$ of participant diagnosed with $\mathrm{TB}$ has Baseline Plasma Viral Load (BPVL) of $>1000$ copies $/ \mathrm{ml}$. Viral rebound was observed in $16.5 \%$ of the study participant. The Proportion of patients having PVL suppressed after taking ART was $72.4 \%$. Study participant contributed 94 person-year of follow up. The incident rate of plasma viral load suppression was $21.8 \%$.

Baseline PVL count in this study is defined as the plasma viral load measured when participant enrolled in the study. At the time of recruitment, $16.6 \%$ of female participants and $7 \%$ of male participants had BPVL < 1000 copies $/ \mu \mathrm{l}$, While $83.3 \%$ of female and $92.9 \%$ of male participants' hade BPVL of $>1000$ copies/ $\mu$ l. The mean and median baseline plasma viral load of study participants were $4.03 \times 10^{5} \mathrm{cells} / \mu \mathrm{l}$ and $2.03 \times 10^{5} \mathrm{cells} / \mu \mathrm{l}$ respectively.

One study participant $(0.4 \%)$ was put on Stavudine (d4T), Lamivudine (3TC), Nevirapine (NVP) regimen, five study participants $(2.1 \%)$ were put on Zidovudine (AZT), 3TC, NVP regimen, 13 study participants (5.3\%) were put on AZT, 3TC, NVP, 13 study participants (5.3\%) was put on AZT, 3TC, Efavirenz (EFV), 17 study participants (7\%) were put on Tenofovir Disoproxil Fumarate (TDF), 3TC, NVP regimen and 168 study participants (69.1\%) were put on TDF, 3TC, EFV.

\section{Bivariate analysis}

Interaction between explanatory variables with the response variable was analyzed by using cross tab features of SPSS, IBM Inc. The baseline PVL status of a patient did not show significant interaction with the event of interest with $x^{2}$ value $2.33(p$-value .127). Other variables which did not show significance includes age of the patient $\mathrm{X}^{2}$ value of 4.44( $p$-value .488), Marital status $\chi^{2}$ value of 6.75 ( $p$-value .081), occupation $\chi^{2}$ value of .383 ( $p$-value .826), education status $\chi^{2}$ value of $1.13(p$ value.770), Mid-Upper Arm Circumference (MUAC) $\mathrm{X}^{2}$ value of .253 (p-value .881 ), BTB with $\chi^{2}$ value of 1.15 
( $p$-value .283). Significant interaction or association with Plasma viral load suppression were observed in sex $\chi 2$ value of 5.06 ( $p$-value 0.024), Body mass index (BMI) with 0.003, BCD4, $x^{2}$ value of 10.98 ( $p$-value 0.004) and ART Treatment regimen with $\chi^{2}$ value of 14.23 ( $p$-value .0 .027$)$.

\section{Comparison between different categories for survival}

Survival distribution for different age groups reveals that there was no significant difference between different age group and other variables in the study in terms of the time to reach suppressed plasma viral load with a logrank test score of $\mathrm{X} 2$ value 0.860 .

The estimated median time to PVL suppression was 181 days (CI: $140.5-221.4$ ) with the age group of 30-39 years having minimum time to achieve suppression with 92 days (CI: 60.1-123.8) and the maximum time required to reach the level was age group between 50 and 59 years.

Significantly different survival distribution curve was observed between different categories of marriage in the study participants. The maximum median time for PVL suppression was observed in unmarried participants with 183 days (CI: 181.7-184.315). The minimum median time registered in the divorced participant category with an estimated median time of 92 days (CI: 91.06-92.93). Significant survival curve difference (log-rank test: $\mathrm{X}^{2}$ value of 8.84 and $p$-value of 0.012 ) was observed in HIV patients with different baseline CD4 count. Median survival time for patients with $<200$ cells $/ \mu$ l measured 182 days (CI: 160.80-203.19), 181 days (142.39-219.60) for BCD4 200-350 cells/ $\mu$ l, 174 days (CI: 133.40-214.59) and 174 days (CI: 133.40-214.59). No significant difference in Virological Failure was observed between different age groups. Virological failure was observed in $27.6 \%$ of the study participants.

\section{Multivariable analysis}

Forward factor selection method (for identifying the synergistic effect of variables on response variables) was used to identify factors that are significantly affected the median time. The Cox-proportional hazard regression method was used to estimate the magnitude of each variable. Variables with a $p$-value of $\leq 0.05$ were included in the model and selected as variables to fit a model that best explains the variance in the equation. Variables identified as significant in this study were marital status with $p$-value 0.023 and baseline CD4 with p-value 0.023 . Educational status ( $p$-value 0.404), MUAC ( $p$-value $0.407)$, BMI ( $p$-value 0.335$)$ and BTB ( $p$-value 0.257$)$ were not found to be associated with viral load suppression. The likelihood ratio test of the fit of the full model relative to the intercept only model is statistically significant $\left(x^{2}=23.14, p=0.027\right)$. This suggests that the model is a significant improvement in model fit relative to the null model. Study participants who are single $(\mathrm{SE}=$ $0.318, p$-value $=0.023)$ and those study participants with $\mathrm{BCD}_{4}<200$ cells $/ \mu \mathrm{l}(\mathrm{SE}=0.0 .288$, $\mathrm{p}$-value $=0.023)$ were a significant positive predictor of the hazard of increase in viral load level beyond suppression level.

\section{Discussion}

The median age of study participants in our study was 32 years' old which is almost similar to the median age of study participants studied by Joseph Davey and et al. in South Africa in 2018 which is 33 years old [9]. The median time for plasma viral load suppression in the study cohort was determined using survival regression analysis. Based on the study, the median time for suppression was 181 days (CI: 140.5-221.4). This finding exactly matches with a study done by the US department of health and human service in 2017, which found the median time between at about 24 weeks after initiation of ART. In another study conducted by Snippenburg W Van, Nellen FJB and Smith C, the median time to undetectable plasma viral load after initiation of treatment was found to be 60 days (12-168 days) which is lower than this study.

As of 2015 , we found that $72 \%$ of study participants achieved viral suppression, but in a study conducted in Brazil, in which study subjects were followed from May 2000 until July 2001, the percentage of HIV patients achieving viral suppression were $62 \%$. This figure was higher in a study conducted in Zimbabwe in 2018 in which viral load suppression in adult was 87 and $93 \%$ in Vietnam in $2016[10,11]$ but similar with the study conducted Cameroon in 2018 which is $72.1 \%$ [12] and slightly lower than viral load suppression rate of $76.8 \%$ in Study conducted in Cambodia in 2018 [13]. 89\% in Uganda studied by Lilian Bulage and et al. in 2017 [14]. This difference could be attributed due to the length of timing of follow up of the patients. A similar study conducted in neighboring Kenya revealed that only 39.85\% of patients had virologocal suppression. In another study conducted by Shikuma CM and et al. the percentage of patients taking ART of different regimens reaching virological suppression after taking ART for 16 weeks showed 93\% viral suppression. In this study, the percent of patients having virological failure was $16.5 \%$. The same study by Shikuma CM and et al., found that $12.2 \%$ of patients have virological failure. Viral suppression rate of this study was found to be similar across different geographical spectrum. Study done in low and middleincome countries of Brazil, Laos people's democratic republic, Malaysia, Mexico, Myanmar and Republic of Moldova have achieved viral suppression of $80 \%$.

Age category of 30-39 years old had minimum time to viral suppression with 92 days (CI: 60.1-123.8) and the maximum time to achieve viral suppression was found 
in the age category of 50-59 years. A significant difference in the proportion of different age groups was not found by log-rank test. A study conducted in Kenya by Cherutich found a significant difference between different age categories in achieving viral suppression level. In this study, there was a significant difference between the age group of 15-29 age groups and 30-64 years' category with a rate of 46.5 and $22.3 \%$ respectively.

A significant difference in plasma viral load suppression was observed in the sex category. Pearson's $\chi^{2}$ for the sex category was 5.06 with a $p$-value of 0.024 . A Significant difference in plasma viral load suppression between sex categories was also achieved in a study conducted by Ballesteros-zebad P. in Mexico, which found a high viral suppression rate in male patients compared to female patients. Other studied which found a significant difference between male and female HIV patient's viral suppression level was done by Pinto $M$. in southern Brazil, and Gray RH., in Uganda. Viral load suppression across the sex category was not significant in a study done by Rangarajan S. Colby DJ in Vietnam. In this study, which is done in Vietnam, there was no significant difference between male and female HIV patients achieving the undetectable viral level. In this study, viral suppression among women was not significantly different with $93.7 \%$ versus $92.9 \%$ in males and females respectively.

The median baseline CD4 was similar to a study conducted in Northern Province, Cameron with 204 cells/ $\mu \mathrm{l}$ and 192 cells $/ \mu$ l respectively. The Survival curve was not significantly different among different BCD4 categories which is also a result supported by the above study mentioned, but similar study in Cameron by Boelaert $\mathrm{M}$. suggested a significant difference in the survival of patients between different BCD4 category.

Baseline Mid upper arm circumference for evaluation of the patient's nutritional status does not appear to be associated with a decrease in viral load suppression. Other studies suggested a different association. A study by Liu E, Spiegelman D, found that baseline lower MUAC was associated with a lower rate of viral suppression from patient plasma. Median baseline plasma viral load of study participants was found to be $2.03 \times 10^{5}$ cells $/ \mu$ l which is higher than the study conducted by Patrick Kazooba in Uganda in 2017, which is $8.1 \times 10^{4}$ cells/ $\mu \mathrm{l}$ and Colin and et al. in Kenya which is $1.6510^{3}$ cells/ $\mu \mathrm{l}[8,15]$.

Body Mass Index affected the survival time of patients with $\chi^{2} 8.98$ and $p$-value of 0.003 . BMI category $<18.5$ $\mathrm{kg} / \mathrm{m} 2$ had increased risk of higher viral replication in a study by Sharma A. and Hoover DR, but the association between obesity and reduced or increased viral load was not observed. No difference in survival curve of HIV patients to reach a viral load level for different ART treatment regimens. Snippenburg W. Van achieved the same curve property in a study, which found no difference between protease inhibitor and non-nucleoside reverse transcriptase inhibitor-based regimens.

At least one instance of viral rebound occurred in $16.5 \%$ of the cohort of HIV patients in the study period. This figure was $45.1 \%$ in the study performed by Guillemi S, Hogg R, Montaner J. equals to the crude incidence of viral rebound of $12.6 \%$ compared to $16.4 \%$ crude incidence of viral rebound in this study. Variables identified as significant in this study were marital status ( $p$-value 0.023) and baseline CD4 ( $p$-value 0.023). Pinto et al., found a significant effect of age and ART usage on the survival of patients taking ART drugs.

Educational status ( $p$-value 0.404), MUAC (p-value 0.407) BMI (p-value 0.335) and BTB (p-value 0.257) has no significant association. There was also no significant association found in a cohort of patients by sex, marital status, educational level, residence or wealth index. Rangarajan S, Colby DJ Gray RH and et al., found a significantly higher viral load after ART treatment in male and concurrent infections with Tuberculosis, Herpes virus, malaria and helmenthiasis. A Study done by Semu H. and Hawkin C. also confirms that baseline MUAC and plasma viral load level does not have significant relation.

\section{Conclusion}

The estimated time to achieve PVL after taking ART was found to be 181 days. Factors affecting time to suppression level were marital status and baseline CD4. Additional factors believed to be influencing the survival curve were also studied, but no significant relation was found with the response variable.

\section{Abbreviations}

3TC: Lamivudine; AFB: Acid fast bacilli; AIDS: Acquired immunodeficiency syndrome; ART: Antiretroviral treatment; BCD4: Baseline cluster of

differentiation 4; BMI: Body mass index; BPVL: Baseline plasma viral load; BTB: Baseline tuberculosis; CD4: Cluster of differentiation 4; d4T: Stavudine; EFV: Efavirenz; FNA: Fine needle aspirate; HIV: Human immunodeficiency virus; IBM: International business machines; L: Löwenstein-Jensen;

MGIT: Mycobacteria growth indicator tube; MTB: Mycobacterium tuberculosis; MUAC: Mid-upper arm circumference; NVP: Nevirapine; PCR: Polymerase chain reaction; PVL: Plasma viral load; SDG: Sustainable development goals; SPSS: Statistical package for the social sciences; TB: Tuberculosis;

TDF: Tenofovir disoproxil fumarate

\section{Acknowledgments}

This work was not possible without due support from Dr. Anton Reepalu, Dr. Taye Tolera Balcha, Dr. Sten Skogmar, Dr. Zelalem Habtamu Jemal, Dr. Erik Sturegård, Dr. Patrik Medstrand and Prof Per Björkman. My academic advisors Tewodros Getinet Yirtaw and Temesgen Geleta also gave me invaluable support to the accomplishment of this work.

\section{Authors' contributions}

My academic advisor TG read revised and approved the whole project starting from title selection through to manuscript preparation and approves the manuscript. JH conceived the project, collect, analysis and interpreted the data and prepares and approves manuscript. JH: Principal Investigator, TG: Academic Advisor. 


\section{Funding}

Funding was secured from Saint Paul's Hospital Millennium Medical College where I earned my MPH. Saint Paul's Hospital Millennium Medical College approved title for use and does not have any role in design of study, collection, analysis, interpretation of data and writing of the manuscript.

\section{Availability of data and materials}

This cohort (longitudinal) study is ongoing starting from 2011 GC until now; cannot deposit the data in the public domain. Data is available at corresponding author upon request. Additional data is available at Lund University, Sweden, contact person, Dr. Anton Reepalu (anton.reepalu@med.lu.se).

\section{Ethics approval and consent to participate}

Institutional review board of Saint Paul's Hospital Millennium Medical College granted an approval of the title in October 09, 2017 (separate file attached to support this narrative) to conduct the project in Oromiya region and in October 12, 2017 Saint Paul's Hospital Millennium Medical College write supporting letter for the project (separate file attached to support this narrative). All Study participants provided written informed consent about their willingness to participation in the project.

\section{Consent for publication}

Not applicable.

\section{Competing interests}

The authors, whose names are listed as J.H. Ali and T.G. Yirtaw declares that we have no affiliation with or involvement in any organization or entity with financial or non-financial interest in the subject matter discussed in the manuscript.

\section{Received: 5 July 2019 Accepted: 11 December 2019}

Published online: 27 December 2019

\section{References}

1. Definition of HIV, CDC. 2015. https://www.cdc.gov/hiv/basics/whatishiv.html

2. Definition of HIV, Mayo Clinic. 2015. http://www.mayoclinic.org/diseasesconditions/HIV-AIDS/basics/definition/con-20013732

3. UNAIDS. An ambitious treatment target to help end the AIDS epidemic 2014.

4. UNAIDS. Focus on location and population, The gap report, UNAIDS, 2014; 2015.

5. Baggaley R, Dalal S, Johnson C, Macdonald V, Mameletzis I, Rodolph M, et al. Beyond the 90-90-90: refocusing HIV prevention as part of the global HIV response. 2016; DHHS. Guidelines for the Use of Antiretroviral Agents in HIV-1 Infected Adults and Adolescents. AIDS info 2017.

6. Ballesteros-Zebad P. Differences in HIV- 1 viral load between males and females antiretroviral- untreated Mexican patients. 2013:44(4):296-301.

7. Reepalu A, Balcha TT, Skogmar S, Jemal ZH, Sturegård E, Medstrand P. High rates of virological suppression in a cohort of human immunodeficiency virus- positive adults receiving antiretroviral therapy in ethiopian health centers irrespective of concomitant tuberculosis. Open Forum Infect Dis. 2014;1(1):ofu039.

8. Kazooba P, Mayanja BN, Levin J, Ben Masiira PK. Virological failure on firstline antiretroviral therapy; associated factors and a pragmatic approach for switching to second-line therapy; evidence from a prospective cohort study in rural South-Western Uganda, 2004-2011. Pan Afr Med J. 2018;29:191.

9. José E, Bello M, Correia AF, Ricardo J, Marins P, Merchan-hamann E, et al. Predictors of virologic failure in HIV/AIDS patients treated with highly active antiretroviral therapy in Brasília, Brazil During 2002-2008. Drug Target Insights. 2008:33-41.

10. Sithole Z, Mbizvo E, Chonzi P, Mungati M, Juru TP, Shambira G. Virological failure among adolescents on ART, Harare City, 2017- a case-control study. BMC Infect Dis. 2018;18(1):469.

11. Rangarajan S, Colby DJ, Le Truong G, Duong BD, Huu HN, Broh TP, et al. Factors associated with HIV viral load suppression on antiretroviral therapy in Vietnam. J Virus Erad. 2016:94-101.

12. Tchouwa GF, Eymard-duvernay S, Cournil A, Lamare N, Serrano L, Butel C, et al. Nationwide estimates of viral load suppression and acquired HIV drug resistance in Cameroon. EClinicalMedicine. 2020;1:21-7. https://doi.org/10. 1016/j.eclinm.2018.06.005.
13. Chhim K, Mburu G, Tuot S, Sopha R, Khol V, Chhoun P, et al. Factors associated with viral non - suppression among adolescents living with HIV in Cambodia: a cross-sectional study. AIDS Res Ther. 2018;15(1):20. https:// doi.org/10.1186/s12981-018-0205-z.

14. Bulage L, Ssewanyana I, Nankabirwa V, Nsubuga F, Kihembo C, Pande G, et al. Factors Associated with virological non-suppression among HIVpositive patients on antiretroviral therapy in Uganda, August 2014 - July 2015. BMC Infect Dis. 2017;17(1):326.

15. Kunzweiler CP, Bailey RC, Mehta SD, Okall DO, Djomand G, Nyunya BO, et al. Factors associated with viral suppression among HIV-positive Kenyan gay and bisexual men who have sex with men. AIDS Care. 2018;30(sup5):S76-88.

\section{Publisher's Note}

Springer Nature remains neutral with regard to jurisdictional claims in published maps and institutional affiliations.
Ready to submit your research? Choose BMC and benefit from:

- fast, convenient online submission

- thorough peer review by experienced researchers in your field

- rapid publication on acceptance

- support for research data, including large and complex data types

- gold Open Access which fosters wider collaboration and increased citations

- maximum visibility for your research: over $100 \mathrm{M}$ website views per year

At BMC, research is always in progress.

Learn more biomedcentral.com/submissions 